\title{
Research on Random Fatigue Load Model of Highway Bridge by Vehicle Traffic Based on GMM
}

\author{
Hao QU, Zhihua NIU, Pingming HUANG
}

\begin{abstract}
Highway bridges have often suffered accidents due to fatigue damage. This paper studies the influence of vehicle operating state on the fatigue performance of bridges. Based on GMM method and K-S test in information statistics, this paper proposes an improved Gaussian hybrid modelling method, and studies the various parameters of vehicle operating state on beam bridge fatigue, such as the impact of the damage and its fatigue life assessment. On this basis, the fatigue cumulative damage formula of multi-vehicle upper bridge is proposed. The traffic load of Shandong JiNan-QingDao expressway has been GMMly analysed by GMM. The Gaussian mixture model is used to fit the vehicle load probability function by standard fatigue vehicle model. Based on the expressway, the vehicle fatigue has been established to facilitate the fatigue load and evaluate the fatigue life. Gradually this paper helps to improve the accuracy and convenience of the probability model, which is conducive to the establishment of a scientific and efficient load probability model for road vehicles.
\end{abstract}

Keywords: fatigue vehicle model; GMM; highway bridges; K-S test, Shandong JiNan-QingDao expressway

\section{FOREWORD}

As an important infrastructure in transportation, bridges play an important role in transportation. The world is actively studying the problem of bridge fatigue [1]. The other is vehicle-related fatigue standards. As China is rich in plateaus, plains, basins etc. that are affected by climate and crust movements, it produced various road bridges [2]. Under these conditions, vehicles also have a greater impact on the fatigue load performance of bridges in various regions. Researchers in China have compiled some specifications by analysing the overall transportation industry, but they are less involved in the analysis of highway bridges [3]. The existing highway reinforced concrete bridge specification lacks rationality $[4,5]$. Specifically, the reinforced concrete bridge has a large scale and the corresponding fatigue research is slow. Under this premise, the reinforced concrete bridge has significant safety and reliability problems. Compared with the international fatigue theory analysis, China's relevant norms are relatively backward, and the actual value is obviously insufficient [6]. The vehicle load of highway bridges only considers the operation situation and directly affects the accuracy of design.

In recent years, with the improvement of China's national strength, the rapid development of modern industrial construction, and the continuous improvement of people's living standards, the demand for transportation has been increasing, resulting in a surge in traffic volume and diversification of automobile axle types. Due to the particularity of our province, which is a coastal city, the load type of the vehicle is compared with that of other provinces [7]. The traffic characteristics not only show a rapid increase in traffic volume, but also a significant trend of heavy load and large-scale. Due to the surge in traffic volume, the proportion of over-limit loaded vehicles has risen sharply $[8,9]$. With the repeated action of the axle load, the cracks continue to increase, which may eventually fail to meet the requirements for engineering accident of safe use. Therefore, a series of problems caused by overlimit overload will endanger the operational safety of bridges and seriously threaten the safety of people's lives [10-12]. It needs to attract the attention of researchers and bridge operation management.

As China's demand for transportation increases sharply, the fatigue of bridges is becoming more frequent. Therefore, the highway bridge components have been subjected to high frequency and strong load amplitude for a long time, so that the fatigue life of the components has been greatly reduced [13]. In this paper, the random fatigue load model of highway bridge vehicles from GMM method is used to analyse the vehicle load parameter statistics and fatigue load spectrum respectively. The mechanical method is used to analyse the distribution of axle load of each axle under different load rates. On this basis, the relationship between different overload rates of representative vehicles and fatigue damage of simple beam bridges is studied. The impact of vehicle operating conditions is studied on the fatigue performance of girder bridges.

\section{RELEVANT THEORETICAL BASIS AND RESEARCH WORK \\ 2.1 GMM for Vehicle Load}

The basic idea of GMM is to represent the color represented by each vehicle load by $M$ states. Usually $M$ is among 3 to 5 , and each state is approximated by a Gaussian distribution.

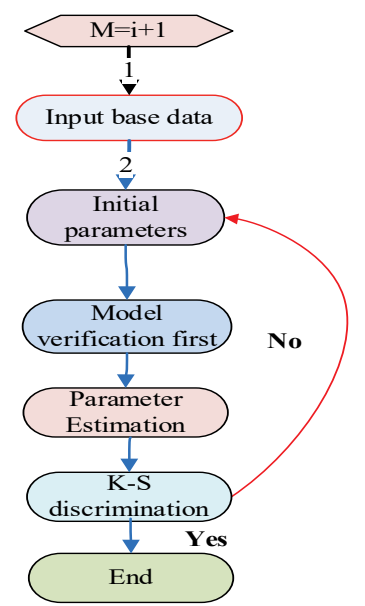

Figure 1 Flow chart of Gaussian mixture model parameter optimization 
The vehicle load represented by sensor is a random variable $X$. At each time $T$, the vehicle load is obtained as a random variable $X$. Then the sensor distribution of vehicle load for the state $k(0<k \leq M)$ can be expressed as follows.

$$
p_{k}\left(x_{t} \mid k, \phi_{k}\right)=\frac{1}{(2 \pi)^{n / 2}\left|\sum_{k}\right|^{1 / 2}} \mathrm{e}^{-\frac{1}{2}\left(x_{t}-\mu_{k}\right)^{T} \sum^{-1}\left(x_{t}-\mu_{k}\right)}
$$

Firstly, EM algorithm is introduced. EM algorithm is a maximum likelihood estimation method for solving distribution parameters from incomplete data or data loss sets. The EM algorithm is usually used to estimate the parameters in the following two cases. A density function $p(x \mid \Phi)$ that depends on the parameter set $\Phi$ and a set of sample data $X=\left(x_{1}, \cdots, x_{N}\right)$ of length $N$ is known. The joint probability density function of the sample data is expressed as follows.

$$
p(x \mid \Phi)=\prod_{i=1}^{N} p\left(x_{i} \mid \Phi\right)=L(\Phi \mid X)
$$

Function $L(\Phi \mid X)$ is called a sample's likelihood function, which is a function of parameter $\Phi$. In the maximum likelihood problem, our goal is to find a maximum value $\Phi^{*}$ for $L$.

$$
\Phi^{*}=\underset{\Phi}{\arg \max } L(\Phi \mid X)
$$

When $p(x \mid \Phi)$ is a single Gaussian distribution, the parameters can be obtained directly by making the derivative $\log (L(\Phi \mid X))$ to be zero. For the mixed Gaussian model, the likelihood function form can be transformed into:

$$
\begin{aligned}
& \log (L(\Phi \mid X))=\log \prod p(x \mid \Phi) \\
& =\sum \log \left(\sum \pi p(x \mid \Phi)\right)
\end{aligned}
$$

This formula contains the logarithm of the sum and is difficult to optimize. However, if we assume that hidden data $Y=\left\{y_{i}\right\}_{i=1}^{N}$ exists, it tells us where density function data are generated from, so that the likelihood function is greatly simplified. Specifically, it is assumed that for each $\mathrm{i}, y_{i} \in 1, \ldots, M$. If the $i$-th sampled data is generated by the density function $k$, then $y_{i}=k$. If you know the value of $Y$, the likelihood function becomes:

$$
\begin{aligned}
& \log (L(\Phi \mid X, Y))=\log (P(X, Y \mid \Phi)) \\
& =\sum_{i=1}^{N} \log \left(P\left(x_{i} \mid y_{i}\right) P(y)\right) \\
& =\sum_{i=1}^{N} \log \left(\pi_{y_{i}} p_{y_{i}}\left(x_{i} \mid \Phi_{y_{i}}\right)\right)
\end{aligned}
$$

For GMM, $p_{i}\left(x \mid \phi_{i}\right)$ known as a Gaussian distribution, the following results can be further derived:

$$
\begin{aligned}
\pi_{i}= & \frac{1}{N} \sum_{l=1}^{N} p\left(i \mid x_{l}, \Phi^{h}\right) \\
\mu_{i}= & \frac{\sum_{l=1}^{N} x_{l} p\left(i \mid x_{l}, \Phi^{h}\right)}{\sum_{l=1}^{N} p\left(i \mid x_{l}, \Phi^{h}\right)} \\
\sum_{i}= & \frac{\sum_{l=1}^{N} p\left(i \mid x_{l}, \Phi^{h}\right)\left(x_{l}-\mu_{i}\right)\left(x_{l}-\mu_{i}\right)^{T}}{\sum_{l=1}^{N} p\left(i \mid x_{l}, \Phi^{h}\right)}
\end{aligned}
$$

These three equations are iterative equations, and the new parameter values are calculated using the sample values and the old parameter values. $\pi_{i}, \mu_{i}$ and $\sum_{i}$ are the newly estimated parameter values, $\Phi^{h}$ is representing the old parameter values, $p\left(i \mid x_{l}, \Phi^{h}\right)$ is indicating the probability that $X$ belongs to the distribution $i$, which can be calculated using the Bayesian formula:

$$
p\left(i \mid x_{l}, \Phi^{h}\right)=\frac{\pi_{i} p\left(i \mid x_{l}, \Phi^{h}\right)}{\sum_{j=1}^{M} \pi_{j} p_{j}\left(x_{l} \mid \Phi^{h}\right)}
$$

The above three equations are parameter estimates derived from known $N$ sample values. For real-time calculations, we derive each expression. For the moment $t$ -1 , the formula is as follows.

$\pi_{k, t-1}=\frac{\sum_{i=1}^{t-1} p\left(k \mid x_{i}, \Phi^{h}\right)}{t-1}$

Then there is at time $t$.

$$
\begin{aligned}
\dot{\pi}_{k, t} & =\frac{\dot{\pi}_{k, t-1} \cdot(t-1)+p\left(k \mid x_{t}, \Phi\right)}{t} \\
& =\left(1-\frac{1}{t}\right) \dot{\pi}_{k, t-1}+\frac{1}{t} p\left(k \mid x_{t}, \Phi\right)
\end{aligned}
$$

\subsection{Vehicle Classification}

The vehicle consists of many different parts. In general, the vehicle as a research object is inevitably difficult to portray because it has no uniform nature. Therefore, multiple parts should be separated first to facilitate separate studies. That is, the type of axle group and the distance among the axle sets are the key factors that determine how the vehicle load is distributed on each axle, which affects the magnitude and many cycles of the stress response when 
the vehicle passes [15]. In this paper, all the vehicles monitored have been divided into 14 types of vehicles. In order to ensure the final establishment of the GMM model, fatigue vehicle load has strong practicability, the model meets one of the following conditions, which will be ignored, more than $90 \%$ of the vehicles weight is less than $30 \mathrm{KN}$. Through the above screening, light vehicles with little impact on fatigue life and rare vehicles with small probability are excluded. However, it is possible to make the modelling object greatly simplify the remaining model as the main body of the fatigue vehicle load.

The measured data of the system is huge and complicated. According to Chinese vehicle classification standards, the models include vehicles, B3 axle medium trucks, 4 axle 4 vehicles and so on. The actual load of each vehicle is different. Firstly, the measured data has been screened and divided. Since the fatigue damage is related to the stress amplitude and the number of cycles, the vehicle load affects the magnitude of the stress amplitude and the number of axles affects the number of cycles of the stress amplitude. Therefore, the key basis for the classification of the vehicle is the vehicle weight and the number of axles [16]. Some models are listed to illustrate the classification method of the vehicle. As shown in Tab. 1 , the 2-axis type has the same number of axles and the probability distribution of the vehicle weight is similar. The 2-axis medium-duty truck has the same number of axles as the former two. The law of heavy probability density distribution is quite different. The probability distribution of the probability density of the 3-axis type vehicle and the 2-axis medium-sized truck is similar but the number of axes is different. Therefore, the former two can only be classified into the same class when the vehicle type is divided. Since the sample number of the 5, 7, 8 and 9 axle vehicles is less than $0.3 \%$, the fatigue damage is limited, so it is not listed separately [17]. According to the weight of the vehicle and the number of axles, the models are classified into five categories as shown in Tab. 1.

Table 1 The axis classification of the vehicle

\begin{tabular}{|c|c|c|c|}
\hline $\begin{array}{c}\text { Vehicle } \\
\text { classification }\end{array}$ & Sketch & Axis classification & $\begin{array}{c}\text { Weight } \\
\text { limit } / \mathrm{t}\end{array}$ \\
\hline 2-axis & Single-single & 20 \\
\hline 3 -axis & $\begin{array}{c}\text { Single-double } \\
\text { 4-axis }\end{array}$ & $\begin{array}{c}\text { Single-single- } \\
\text { double }\end{array}$ & 40 \\
\hline 5-axis & $\begin{array}{c}\text { Single-single- } \\
\text { three }\end{array}$ & 50 \\
\hline 6-axis & $\begin{array}{c}\text { Single-double- } \\
\text { three }\end{array}$ & 55 \\
\hline
\end{tabular}

According to the above specifications and actual conditions, this paper makes the following provisions for the maximum total weight limit: 18 tons for the first type of vehicle, 25 tons for the second type, 35 tons for the third type, and 43 for the fourth type. The fifth category of vehicles takes 49 tons, and the vehicles of the fifth category have more than six axles. For each additional axle, the maximum total weight limit is 10 tons.

\subsection{Literature Review}

The traffic survey of highway bridges in China started relatively late. According to statistics, in 1988, statistics are vehicleried out on two observatories in Qiuzhuang and Wuqiao County, Daqing, Wuqing County, Tianjin, and the vehicle overload rate of the Jinuo Expressway was 40\%. In 2012, a dynamic weighing system was set up on five typical expressways in Guangzhou. The vehicle load model has been obtained through field survey data analysis. At the same time, the actual bridge load effect, the highway bridge culvert specifications have been compared, and the actual load has been obtained. The effect is about 1.4 times the design value. In 2012, Mustafa proposed a research on the vehicle load model of the Beijing-Shanghai Expressway (G2 > Xinqiaohe Bridge) based on the dynamic weighing system, established the probability distribution model under the actual operating state of the vehicle, and obtained the general operating status of the vehicle [18]. The intensive operation states are single-peak distribution and multi-peak distribution respectively, and the probability distribution of the driving distance is subject to the Weibull distribution. The left and right bridge loads are 1.06-1.34 times and 1.6-1, respectively 69 times, it can be seen that the right-hand vehicle has a serious overload rate.

In view of the increasingly serious over-loading phenomenon, the analysis of the damage caused by vehicle overload on highway bridge and culvert structure and the problem of overloading and over-limit of vehicle handling has become a hot-spot problem in recent years, as well as the random fatigue load model of highway bridge vehicles. Liang [19] studied the fatigue reliability of orthotropic bridge decks with long-span cable-stayed bridges under consideration of vehicle and wind coupling. Niu Yutao [20] combined the vehicle monitoring data with the reliability evaluation of the Runyang Yangtze River Bridge random finite element model to show the results of FEM and SHM. Niu Yutao. (2018) obtained the reinforced degraded specimens in corrosive environment by wet salt sand rusting method, selected the fatigue stress level of highway bridges under the load of vehicles, and analysed the corroded reinforced concrete bars through the bending fatigue test of 8 corroded reinforced beams [20]. Based on theoretical analysis of sufficient mixing numbers, the diversified models and the load factor of different vehicles form a very complex vehicle load. Chen et al., [21] prove that the Gaussian mixture model is gradually approaching the probability density distribution function, which can be described as a Gaussian mixture model in the probability of distributed vehicle load samples. For the multimodal hybrid model, the class label for training known samples is seriously insufficient or cannot be observed, and it is considered a typical incomplete evaluation data operation. EM algorithm can scientifically deal with the typical incomplete data likelihood problem. Shunlong Li et al. proposed a Gaussian Mixture Model (GMM). Based on the vehicle dynamic weighing data, the equivalent fatigue vehicle load of Expressway was established based on the equivalent fatigue principle [22]. The test results show that the fatigue failure mode of the corroded beam is mainly brittle fracture. L. Tian et al., (2018) study the probability model of the load of a large bridge vehicle, select the monitoring data of the Nanxi Yangtze River Bridge based 
on the dynamic weighing system (WIM) [23]. The model parameters are estimated by nonlinear least squares estimation. Compared with the foreign fatigue design theory, China is still in its infancy, and lacks comprehensive vehicle full load statistics, which leads to the relevant specifications for the fatigue design of highway bridges. However, the standard fatigue vehicles at home and abroad are different, and the Anglo-American standard fatigue vehicles cannot be a very good description of the current vehicle operation situation in China. In view of the situation, this paper uses the Gaussian mixture model to study the random fatigue load model of highway bridge vehicles.

\section{RANDOM FATIGUE LOAD MODEL OF HIGHWAY BRIDGE VEHICLES BASED ON GMM \\ 3.1 Detection Object}

This paper selects the data about vehicle traffic obtained by the Zibo Expressway toll station, which provides fatigue load spectrum analysis. The layout is shown in Fig. 2. The Zibo expressway is mainly composed of three parts: the basic road section, the interweaving road section, the ramp road, and the ramp connection point. The traffic flow of Zibo expressway is completely continuous, without any external factors, but in the process of entering and exiting, the traffic flow still has the phenomenon of confluence, diversion, and interweaving [24]. In this paper, the finite element modeling analysis of the bridge upper structure has been vehicleried out by the bridge professional finite element software Midas/ Civil 2015.

The Ziboexpressway has four or more lanes and is equipped with a central separation belt. All are threedimensionally intersected, and have perfect traffic safety facilities and management facilities. All of them have been controlled for access and designed for high-speed vehicles.

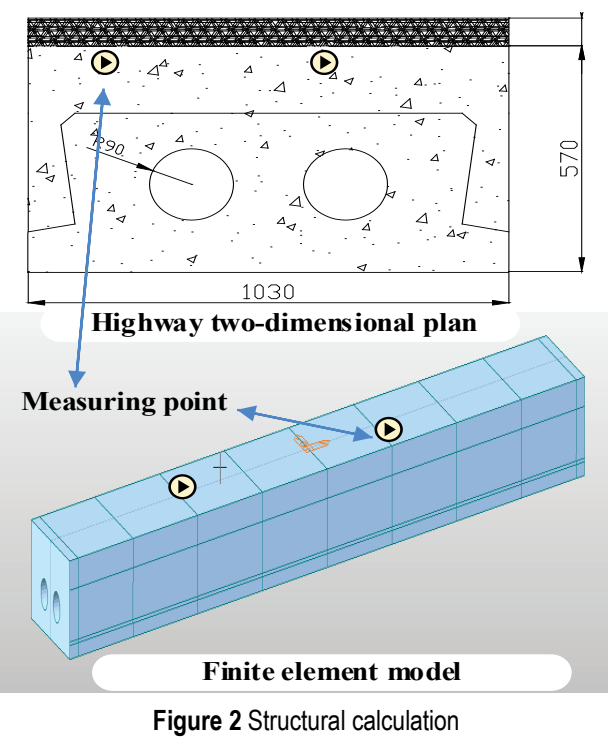

\subsection{Standard Fatigue Vehicle Modeling 3.2.1 3-Axle Trucks for Loading}

The test uses 4 front, rear and eight rounds of a total of 3 axle trucks for loading. The general outline of the loading truck is shown in Fig. 3. The axle load and wheelbase data are shown in Tab. 2.
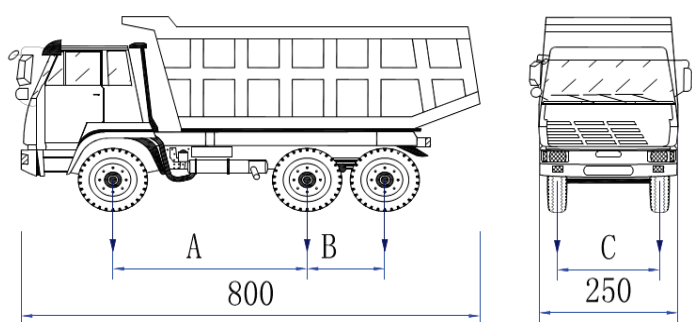

Figure 3 Schematic diagram of a common 3-axle truck

Table 2 Axle load and wheelbase data of common 3-axle trucks

\begin{tabular}{|c|c|c|c|c|c|c|c|}
\hline \multirow{2}{*}{ NO. } & \multicolumn{4}{|c|}{ Vehicle weight / t } & \multicolumn{3}{|c|}{ Wheelbase and track / m } \\
\hline & Rear axle & middle axle & Front axle & Total & Front-middle (A) & Middle-rear (B) & Track (C) \\
\hline 1 & 14.2 & 14.2 & 7.4 & 35.8 & 3.95 & 1.35 & 1.8 \\
\hline 2 & 14.0 & 14.0 & 7.2 & 35.2 & 3.95 & 1.35 & 1.8 \\
\hline 3 & 14.1 & 14.1 & 6.6 & 34.8 & 3.95 & 1.35 & 1.8 \\
\hline 4 & 14.2 & 14.2 & 6.7 & 35.1 & 3.95 & 1.35 & 1.8 \\
\hline
\end{tabular}

\subsubsection{Vehicle Weight}

Taking the 6-axis vehicle as an example, the method of establishing the vehicle weight GMM model is explained. The distribution of the weight of the 6-axis vehicle shows that the vehicle weight of the model has obvious bimodal distribution characteristics [25]. In general, resorting to a mixed distribution model can ideally solve the fitting problem of multimodal probability density functions. If a population consists of $\mathrm{n}$ children, the mixing ratio and probability density function of the corresponding children are $\alpha_{1} \ldots, \alpha_{n}\left(\sum_{i=1}^{n} \alpha_{i}=1\right)$ and $f_{1}(t), \ldots, f_{n}(t)$, Then the overall probability density function is $f(t)=\alpha_{1} f_{1}(t)+\cdots+\alpha_{n} f_{n}(t) \cdot f(t)$ is a general form of a mixed distribution model characterized by the number of children $\mathrm{n}$ and the distribution class of the child, if $f_{i}(t), i=1, \ldots, n$ is a normal distribution, it is called $f(t)$ The mixed distribution described is a remixed Gauss distribution.

The research object here is the weight of a single model, so the possibility of a double-peak distribution caused by the vehicle including multiple load levels is excluded. Because the vehicle weight distribution of light vehicles has no double peak phenomenon, the vehicle weight distribution of the heavy vehicles has a bimodal characteristic. Firstly, it is assumed that the weight of the 6-axis vehicle includes 2 sub-body, namely the empty load and the full load, and the probability density function fitted by the double-mixed distribution model can reflect the basic trend of the 6-axle vehicle redistribution. However, in several parts, especially in the transition zone between the two distribution peaks, the empirical density of the measured data is quite different. Therefore, it is assumed that the six-axle vehicle weight is generally composed of 
three sub-body weights: empty load weight, half load weight, and full load weight. That is, using three subdistributions to fit the probability density function of the double peaks. Selecting the triple-mixed Gauss distribution to fit the probability density function of the vehicle weight, then

$$
f(x)=\sum_{i=1}^{3} \alpha_{i} f_{i}(x)
$$

Corresponding to the mixed Gauss distribution model.

$f_{i}(x)=\frac{1}{\sqrt{2 \pi \sigma_{i}^{2}}} \exp \left[-\frac{\left(x-\mu_{i}\right)^{2}}{2 \sigma_{i}^{2}}\right]$

In the formula, the weight parameter is $\alpha_{i}, \mu_{1}, \ldots, \mu_{n}$ expresses the mean value of the normal distribution child; $\sigma_{1}^{2}, \ldots, \sigma_{n}^{2}$ means the variance of the normal distribution child, There are many ways to solve the mixed model parameters. The EM algorithm is the most common and effective algorithm. Practice shows that for the parameter estimation problem of hybrid distribution model, the application of EM algorithm can achieve satisfactory results.

\subsection{Data Source}

This topic is derived from the project "Study Group on Fatigue Test in Jinan-Qingdao High-speed Railway of Shandong Province". The data is selected from the data of Zibo Highway exit Toll Station in Jinan Qingdao Expressway. The time is from January 1, 2013 to January 1,2016 . The data of 6400008 vehicles, vehicle weight and axle load recorded in the vehicle weighing data at the entrance of Zibo Expressway. Bridge and structure load test system using equipment $R S-Q L 06 E$ made in Japan. Dynamic strain acquisition and analysis system uses instrumentsINV3060S made in the United States. Dynamic strain sensor is HY-65DJB3000B by England. Since the load of the overloaded over-limit vehicle far exceeds the design load of the road and the bridge, the road surface is damaged and the bridge is broken, and the service life is greatly shortened. Usually the highway load design is much larger than 3T. Since vehicles with a weight of less than $3 \mathrm{t}$ have little effect on the fatigue damage of the bridge such vehicles can be ignored and meet the British BS5400 specification. Therefore, the types of vehicles that can ignore the effects of fatigue damage on bridges are listed in Tab. 3. The total number of vehicles after the number of vehicles in Tab. 3 is 2169587.

Table 3 Axis classification of Jinan-Qingdao high-speed vehicles

\begin{tabular}{|c|c|c|c|c|}
\hline Axis type & NO. & Type & NO. & Ratio \\
\hline 2 & 712092 & $1-1$ & 712090 & $100 \%$ \\
\hline \multirow{2}{*}{3} & \multirow{2}{*}{131010} & $1-2$ & 19123 & $15 \%$ \\
\hline & & $1-1-1$ & 111887 & $85 \%$ \\
\hline \multirow{4}{*}{4} & \multirow{4}{*}{121308} & $1-1-1-1$ & 3667 & $3 \%$ \\
\hline & & $1-3$ & 46 & $0.1 \%$ \\
\hline & & $1-2-1$ & 356 & $0.3 \%$ \\
\hline & & $1-1-2$ & 117239 & $96.6 \%$ \\
\hline \multirow{7}{*}{5} & \multirow{7}{*}{143834} & $1-1-1-1-1$ & 5812 & $4 \%$ \\
\hline & & $1-2-1-1$ & 384 & $0.3 \%$ \\
\hline & & $1-1-2-1$ & 998 & $0.7 \%$ \\
\hline & & $1-1-1-2$ & 2490 & $4 \%$ \\
\hline & & $1-3-1$ & 33 & $1.8 \%$ \\
\hline & & $1-2-2$ & 7546 & $5.2 \%$ \\
\hline & & $1-1-3$ & 126571 & $88 \%$ \\
\hline \multirow{7}{*}{6} & \multirow{7}{*}{1061345} & $1-1-1-1-1-1$ & 20511 & $1.9 \%$ \\
\hline & & $\begin{array}{l}1-2-1-1-1 \\
1-1-2-1-1 \\
1-1-1-2-1 \\
1-1-1-1-2\end{array}$ & 8122 & $0.8 \%$ \\
\hline & & $\begin{array}{l}1-2-2-1 \\
1-2-1-2 \\
1-3-1-1\end{array}$ & 4818 & $0.5 \%$ \\
\hline & & $\begin{array}{l}1-1-2-2 \\
1-1-3-1\end{array}$ & 1837 & $0.2 \%$ \\
\hline & & $1-3-2$ & 78 & $0 \%$ \\
\hline & & $1-1-1-3$ & 445922 & $42 \%$ \\
\hline & & $1-2-3$ & 580057 & $54.6 \%$ \\
\hline
\end{tabular}

For the sake of analysis, this paper describes a vehicle according to the position of the single shaft or the coupling from the front to the rear of the vehicle, which is called a shaft type. For example, a 3-axis vehicle with a front single-axle rear twin-axle can be called a "1-2 vehicle"; for example, a 6-axle vehicle with a front single-shaft, a double-shaft, and a rear triple-link can be called "1-2 -3 type vehicle", and so on, the vehicle axle types listed in this paper are listed in Tab. 3.

According to the above content, the shaft type, quantity, and percentage of vehicles that can cause bridge fatigue are shown in Tab. 3. It can be seen that the total number of vehicles that can cause bridge fatigue (referred to as fatigue vehicles) is $2,120,435$ vehicles, accounting for the total number of vehicles. $33.1 \%$.

\subsection{Fatigue Load Spectrum of Zibo Expressway}

For the vehicle weight distribution of a single vehicle, due to the different load conditions such as full load, no 
load, and half load, the general Gaussian distribution, logarithmic distribution and other models cannot be used for parameter estimation. Therefore, the Gaussian mixture model in statistics is introduced [26]. Taking the 6-axis vehicle as an example, the vehicle weight distribution is shown in Fig. 4.
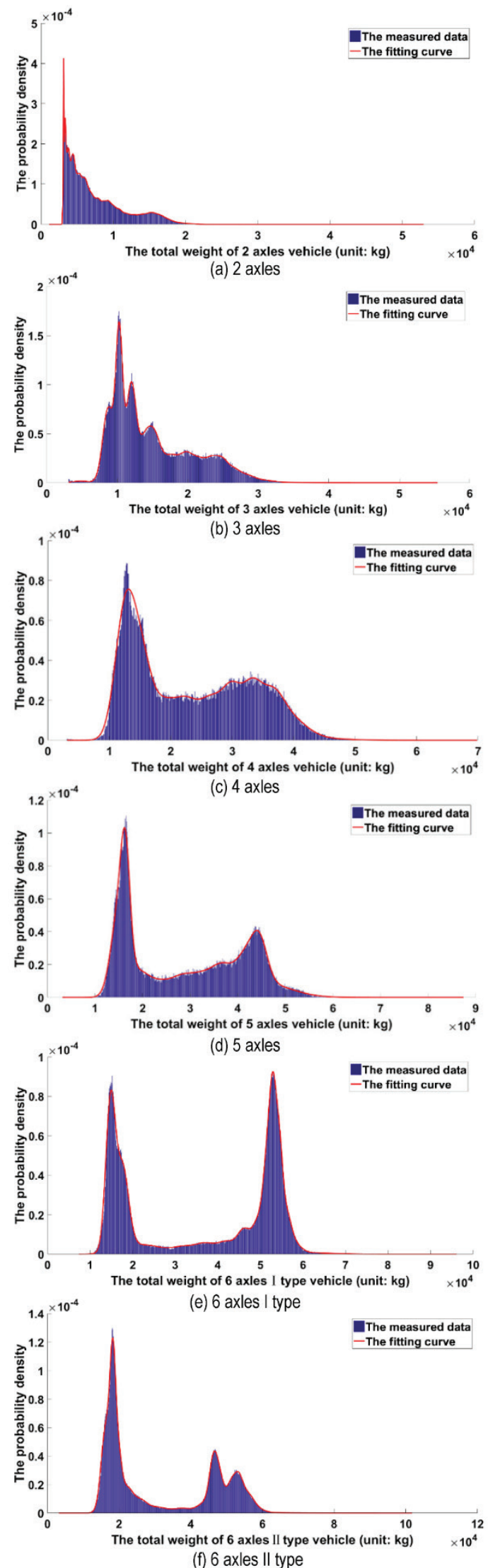

Figure 46 kinds of axle weight data between measures data and GMM

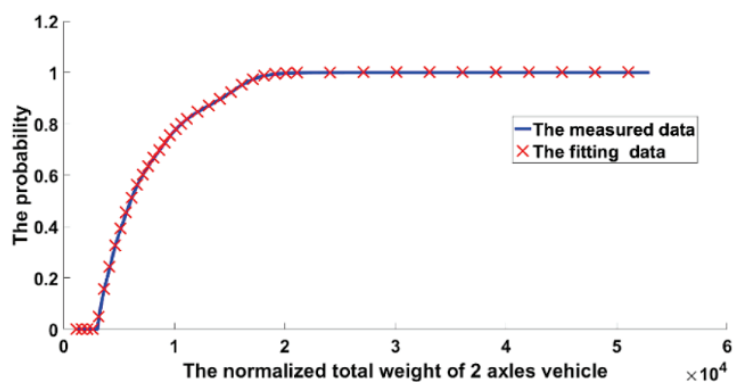

(a) 2 axles

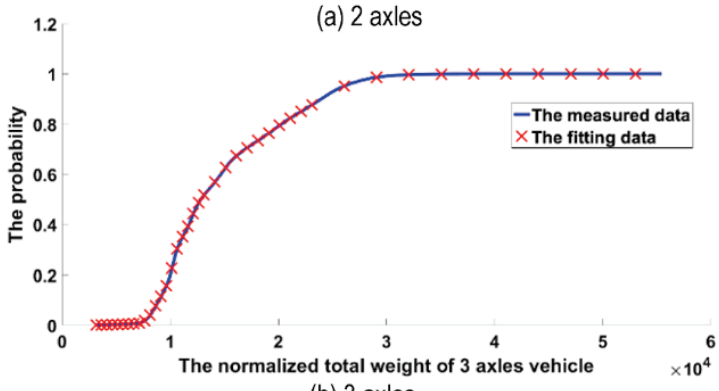

(b) 3 axles

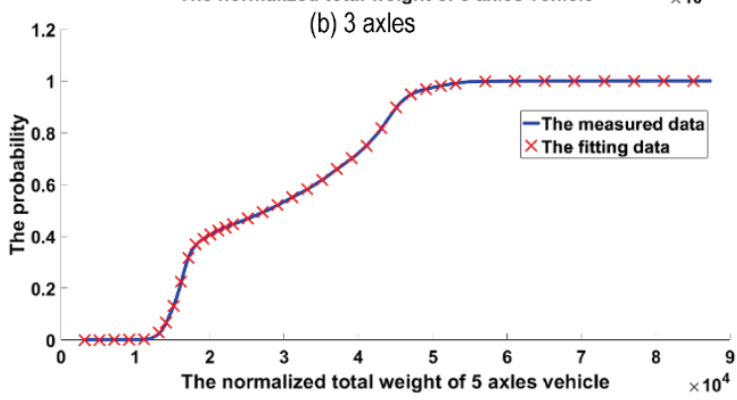

(c) 5 axles
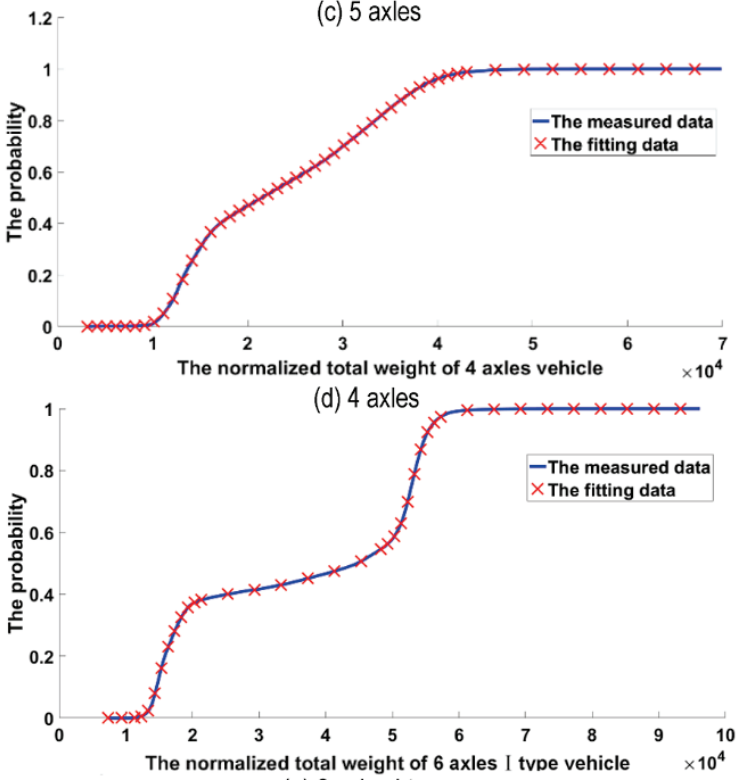

(e) 6 axles I type

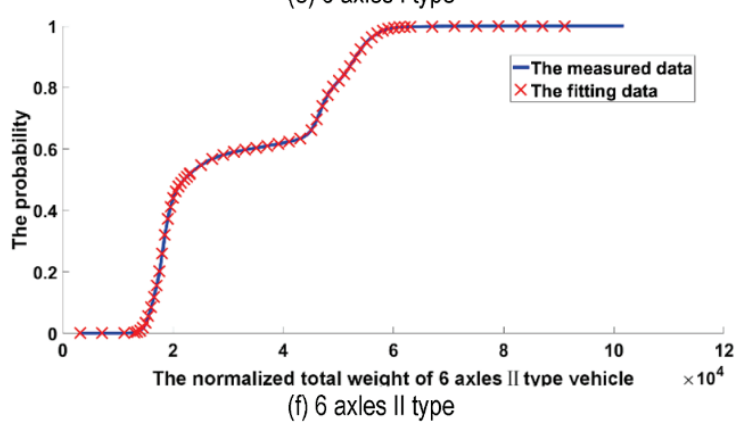

Figure 5 the cumulative probability for 6 kinds of axle weight data between measures data and GMM

Fig. 4 shows the typical freight vehicles and monthly 
traffic volume of Zibo Expressway. And there are more over-limit vehicles, accounting for most of the total monthly traffic of the same model. The first 1-axis obeys a unimodal distribution, and the probability of occurrence of a peak region on 1 -axis is $4 \times 10^{-4}$. For multi-axis models, the two peak probability intervals are between $1.2 \times 10^{-4}$ and $0.3 \times 10^{-4}$.

Since the distribution of vehicle weight is greatly affected by the lane and time zone, the vehicle weight distribution in each lane is compared, and the vehicle weight distribution in the same lane is compared in the longitudinal direction. The horizontal comparison of the cumulative probability density of each lane and the cumulative probability density of the longitudinal comparison lanes in the 1-minute period are shown in Fig. 5 .

The proportion of different vehicle types in the main traffic volume, because the vehicle weight is relatively small compared to other trucks, you can ignore its fatigue damage to the bridge. In this paper, the total weight of the vehicle model and the weight of each axis are statistically analyzed, and the probability density cube map is obtained using numerical software. The probability density histogram is numerically fitted with a Gaussian mixture model to finally obtain its probability density distribution function. From Fig. 5, it is found that the 2 axis and 3 axis are the smoothest, directly hit the maximum probability density, and enter the fatigue state fastest. The other multiaxis has two probability densities, and it enters a fatigue state after a buffer time.

\subsection{Fatigue Load Spectrum Analysis}

The load is divided into two categories: static load and dynamic load. Due to the uncertainty of random loads, this spectrum cannot be used directly and must be GMM processed. The processed load-time-history is called the load spectrum. The load spectrum is a graph with GMM properties that can essentially reflect the load changes of the part.

\subsubsection{Fatigue Load Spectrum}

In this paper, the GMM analysis of vehicle load parameters, the distribution type and GMM law of load parameters of each vehicle are obtained, but the actual project needs a more direct expression. This section combines the K-S test and the linear cumulative damage law to simplify the distribution model. The principle of simplification is to assume that the fatigue damage caused by the $n$ model vehicles to the structural system is the same as that of the $n$ equivalent model vehicles.

\subsubsection{Loading Condition and Observation Section}

According to the requirements of appearance inspection, on-site test convenience, contract and bidding documents, the first span, and the second span are the test spans of this load test, and the test is vehicleried out in three working conditions. The specific load conditions and observation sections are as follows.

Strain measurement point marked in Fig. 2, which we can see. At each observation section, the measurement points are arranged at the bottom plate of each girder and part of the web position. The layout is shown in Fig. 6 . Deflection point: The deflection point has been arranged at the center of each beam of the A-A and C-C sections, and the settlement point is arranged at the top of the pier. The Measured time domain curve and frequency domain curve are shown in Fig. 6.

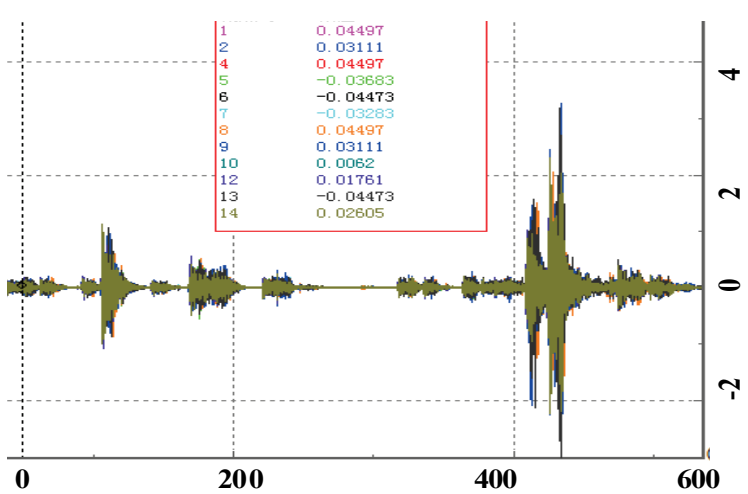

(a) Time domain curve

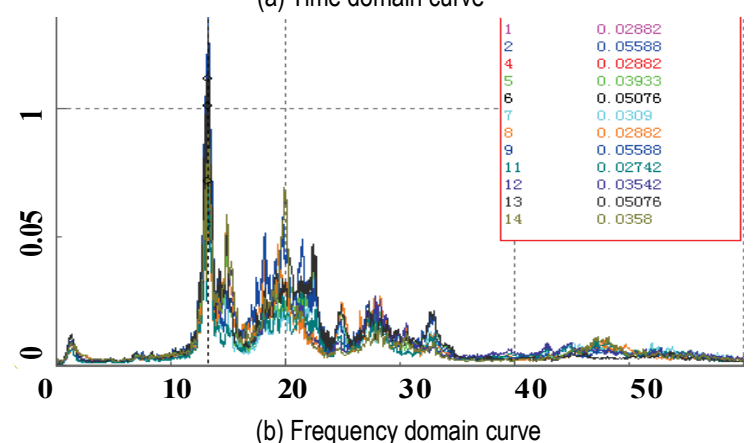

Figure 6 Measured time domain curve and frequency domain curve

The K-S test is a method used in GMM analysis of a set of data in statistics. It is a method of comparing the data that needs to be GMM analysed with another set of standard data to find the deviation between it and the standard data. Generally, in the K-S test, the cumulative distribution function of the two sets of observation data to be compared is first calculated, and then the maximum value $\mathrm{D}$ among the absolute values of the differences of the two cumulative distribution functions is obtained. In this paper, the K-S check is performed on the maximum frequency for Fig. 6, and the measurement points of the high-speed pavements $1 \#, 2 \#, 3 \#$ and $4 \#$ are selected for verification.

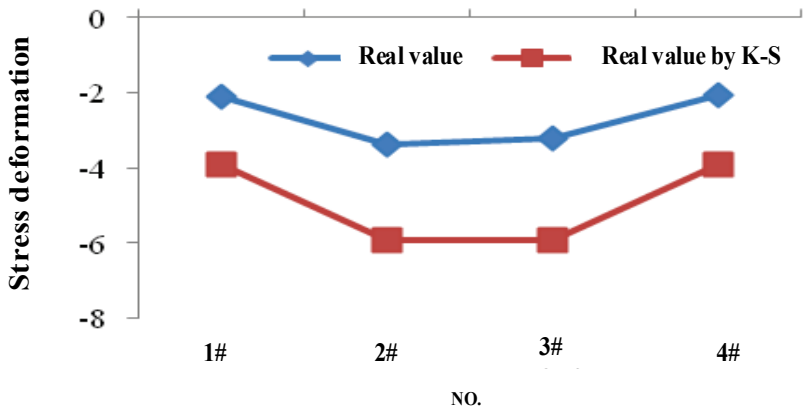

Figure 7 Reinforcement stress curve of mid-bottom edge of C2-C3 standard fatigue vehicle crossing bridge

The measured time course and frequency domain curves are shown in Fig. 7 below. Finally, a table lookup is 
made to determine if the fatigue $D$ value falls within the required confidence interval. If the $D$ value falls within the corresponding confidence interval, it indicates that the detected data meets the requirements.

\section{CONCLUSION}

In this paper, the influence of vehicle operating state on the fatigue performance of highway bridges has been taken as the starting point. The influence of various parameters of vehicle operating state on the fatigue damage of beam bridges is studied. At the same time, the traffic load of Jinan-Qingdao Expressway Zibo section has been investigated and analyzed Fatigue life assessment. The relationship between the axle load and the axle load of different axles is calculated. Based on this, the relationship between vehicle overload rate and beam-bridge fatigue damage is derived. The speed of the vehicle, the distance between the train and the span of the bridge are analyzed. Based on this, the fatigue cumulative damage formula of multi-vehicle upper bridge is proposed. The traffic load statistics of Jinan-Qingdao Expressway is calculated by Gaussian mixture model, and the vehicle load probability function is fitted by Gaussian mixture model. The fatigue reliability of analysis framework of Reinforced concrete Highway Bridge considering traffic volume is established.

The main results of paper innovations are clarified as follows. This paper adopts random fatigue load model of Highway Bridge by vehicle traffic based on GMM. The KSanalysis results show the feasibility of the method. In addition to traffic volume and axle weight, parameters such as vehicle speed and road surface flatness also have a certain impact on the fatigue stress analysis of Reinforced concrete highway Bridge. In addition, the results of this paper are subject to further reconciliation with stress data based on long-term health monitoring. It can be applied to build fatigue vehicle load models under various traffic conditions or provide a reference.

\section{REFERENCES}

[1] Cui, C., Zhang, Q., Luo, Y., Hao, H., \& Li, J. (2018). Fatigue reliability evaluation of deck-to-rib welded joints in osd considering stochastic traffic load and welding residual stress. International Journal of Fatigue, 111. https://doi.org/10.1016/j.ijfatigue.2018.02.021

[2] Loh, P. R., Tucker, G., Bulik-Sullivan, B. K., Vilhjalmsson, B. J., Finucane, H. K., Salem, R. M. et al. (2015). Efficient Bayesian mixed-model analysis increases association power in large cohorts. Nature genetics, 47(3), 284. https://doi.org/10.1038/ng.3190

[3] Liu, Z., Zhou, L., Leung, H., \& Shum, H. P. (2016). Kinect posture reconstruction based on a local mixture of gaussian process models. IEEE transactions on visualization and computer graphics, 22(11), 2437-2450. https://doi.org/10.1109/TVCG.2015.2510000

[4] Gungor, O. E., Al-Qadi, I. L., \& Mann, J. (2018). Detect and charge: Machine learning based fully data-driven framework for computing overweight vehicle fee for bridges. Automation in Construction, 96, 200-210. https://doi.org/10.1016/j.autcon.2018.09.007

[5] Haar, C. V. D. \& Marx, S. (2018). A strain model for fatigueloaded concrete. Structural Concrete, 19(2), 463-471. https://doi.org/10.1002/suco.201700029

[6] Varma, V. \& Rajamohan, V. (2019). Enhancement of low cycle vibration induced fatigue life of composite beam having central hole using cnt reinforcement: an experimental study. Mechanics of Advanced Materials and Structures, 19. https://doi.org/10.1080/15376494.2018.1541489

[7] Nariman, N. A. (2018). Thermal fluid-structure interaction and coupled thermal-stress analysis in a cable stayed bridge exposed to fire. Frontiers of Structural \& Civil Engineering, 12(4). https://doi.org/10.1007/s11709-018-0452-z

[8] Liu, Y., Zhang, H., Liu, Y., Deng, Y., Jiang, N., \& Lu, N. (2017). Fatigue reliability assessment for orthotropic steel deck details under traffic flow and temperature loading. Engineering Failure Analysis, 71, 179-194. https://doi.org/10.1016/j.engfailanal.2016.11.007

[9] Zhu, J. \& Zhang, W. (2018). Probabilistic fatigue damage assessment of coastal slender bridges under coupled dynamic loads. Engineering Structures, 166, 274-285. https://doi.org/10.1016/j.engstruct.2018.03.073

[10] Skakun, S., Franch, B., Vermote, E., Roger, J. C., BeckerReshef, I., Justice, C., \& Kussul, N. (2017). Early season large-area winter crop mapping using MODIS NDVI data, growing degree days information and a Gaussian mixture model. Remote sensing of environment, 195, 244-258. https://doi.org/10.1016/j.rse.2017.04.026

[11] Hasni, H., Alavi, A. H., Chatti, K., \& Lajnef, N. (2017). A self-powered surface sensing approach for detection of bottom-up cracking in asphalt pavements: theoretical/numerical modeling. Construction and Building Materials, 144, 728-746. https://doi.org/10.1016/i.conbuildmat.2017.03.197

[12] Yan, D., Luo, Y., Lu, N., Yuan, M., \& Beer, M. (2017). Fatigue Stress Spectra and Reliability Evaluation of Short-to Medium-Span Bridges under Stochastic and Dynamic Traffic Loads. Journal of Bridge Engineering, 22(12), 04017102 . https://doi.org/10.1061/(ASCE)BE.1943-5592.0001137

[13] Li, F. \& Xu, J. (2015). A new prognostics method for state of health estimation of lithium-ion batteries based on a mixture of Gaussian process models and particle filter. Microelectronics Reliability, 55(7), 1035-1045. https://doi.org/10.1016/j.microrel.2015.02.025

[14] Chen, Z. W. (2014). Fatigue Reliability Assessment of Multiloading Suspension Bridges Based on SHMS. Engineering Mechanics, 31(7), 99-105.

[15] Guot, Frangopold, M., \& Cheny (2012). Fatigue Reliability Assessment of Steel Bridge Details Integrating Weigh in motion Data and Probabilistic Finite Element Analysis. Computers \& Structures, 112-113, 245-257. https://doi.org/10.1016/j.compstruc.2012.09.002

[16] Guilherme, S. A., Gonçalo, F., Abilio, M.P de Jesus, \& Calçada, R. (2018). Fatigue assessment of a high-speed railway composite steel-concrete bridge by the hot-spot stress method. International Journal of Structural Integrity, 9(3). https://doi.org/10.1108/IJSI-11-2017-0061

[17] Sun, X., Wang, H. (2015). Study on Fatigue Damage of Serviced Reinforced Bridges under Overload Operation. Journal of Applied Basic and Engineering Sciences, 16(5), 733-740.

[18] Aygül, M., Al-Emrani, M., \& Urushadze, S. (2012). Modelling and fatigue life assessment of orthotropic bridge deck details using fem. International Journal of Fatigue, 40, 129-142. https://doi.org/10.1016/j.jfatigue.2011.12.015

[19] Weiquan, L. (2016). Fatigue performance analysis of highway steel bridges under fatigue load model III. Urban road bridges and flood control, (7), 129-132.

[20] Jutao, N. (2018). Experimental study on fatigue performance of rust-damped highway bridges based on vehicle load. Journal of Civil Engineering, (3).

[21] Chen, B., Ye, Z. N., Chen, Z., \& Xie, X. (2018). Bridge vehicle load model on different grades of roads in china based on weigh-in-motion (wim) data. Measurement, 
S0263224118301751

https://doi.org/10.1016/j.measurement.2018.03.005

[22] Li, S., Wei, S., Bao, Y., \& Li, H. (2018). Condition assessment of cables by pattern recognition of vehicleinduced cable tension ratio. Engineering Structures, 155, 115. https://doi.org/10.1016/j.engstruct.2017.09.063

[23] Tian, L., Xu, J., Jia, X., \& Fang, J. (2018). Experimental research on horizontal curve alignment indexs of highway in high-altitude area based on driver fatigue. Journal of Nanjing University of Science \& Technology, 42(3), 370-373 and 379

[24] Gao, R., Li, J., \& Ang, A. H. S. (2019). Stochastic analysis of fatigue of concrete bridges. Structure and Infrastructure Engineering, 15(7), 925-939. https://doi.org/10.1080/15732479.2019.1569073

[25] Zhang, J. \& Au, F. (2016). Fatigue reliability assessment considering traffic flow variation based on weigh-in-motion data. Advances in Structural Engineering. https://doi.org/10.1177/1369433216646011

[26] Luo, Y., Yan, D., \& Yuan, M. (2018). Fatigue reliability evaluation of short-span concrete bridges under dynamic impacts of stochastic truck loading. International Journal of Reliability and Safety, 12(1-2), 103-121. https://doi.org/10.1504/IJRS.2018.092514

\section{Contact information:}

\section{Hao QU}

(Corresponding author)

School of Highway, Chang'an University,

Xi'an, Shaanxi Province, 710064, China

E-mail: quhao1987@yeah.net

\section{Zhihua NIU}

Power China Northwest Engineering Corporation Limited,

Xi'an, Shaanxi Province, 710065, China

\section{Pingming HUANG}

School of Highway, Chang'an University,

Xi'an, Shaanxi Province, 710064, China 